\title{
General criteria for asymptotic and exponential stabilities of neural network models with unbounded delays
}

\author{
Teresa Faria ${ }^{a 1}$ and José J. Oliveira ${ }^{b}$ \\ ${ }^{a}$ Departamento de Matemática and CMAF, Faculdade de Ciências, \\ Universidade de Lisboa, Campo Grande, 1749-016 Lisboa, Portugal \\ e-mail: tfaria@ptmat.fc.ul.pt \\ ${ }^{b}$ Departamento de Matemática e Aplicações and CMAT, Escola de Ciências, \\ Universidade do Minho, Campus de Gualtar, 4710-057 Braga, Portugal \\ e-mail: jjoliveira@math.uminho.pt
}

\begin{abstract}
For a family of differential equations with infinite delay, we give sufficient conditions for the global asymptotic, and global exponential stability of an equilibrium point. This family includes most of the delayed models of neural networks of Cohen-Grossberg type, with both bounded and unbounded distributed delay, for which general asymptotic and exponential stability criteria are derived. As illustrations, the results are applied to several concrete models studied in the literature, and a comparison of results is given.
\end{abstract}

Keywords: Cohen-Grossberg neural network, infinite delay, distributed delay, global asymptotic stability, global exponential stability.

2010 Mathematics Subject Classification: 34K20, 34K25, 92B20.

\section{Introduction}

Since the pioneering work of Hopfield in 1982 [14], several classes of neural network models have been the subject of an active research, due to their large application in a variety of scientific areas, such as combinatorial optimization, content-addressable memory, pattern recognition, signal and image processing, associative memory.

In 1983, Cohen and Grossberg [3] proposed and studied the artificial neural network described by a system of ordinary differential equations (ODEs),

$$
\dot{x}_{i}=-k_{i}\left(x_{i}\right)\left[b_{i}\left(x_{i}\right)-\sum_{j=1}^{n} a_{i j} f_{j}\left(x_{j}\right)\right], \quad i=1, \ldots, n,
$$

and in 1984 Hopfield [15] studied the particular situation of (1.1) with $k_{i} \equiv 1$,

$$
\dot{x}_{i}=-b_{i} x_{i}+\sum_{j=1}^{n} a_{i j} f_{j}\left(x_{j}\right), \quad i=1, \ldots, n .
$$

In order to be more realistic, differential equations describing neural networks should incorporate time delays to take into account the synaptic transmission time among neurons, or, in artificial neuron networks, the communication time among amplifiers. In 1989, Marcus and Westervelt [20] introduced for the first time a discrete delay in the Hopfield model (1.2), and observed that the delay can destabilize the system; it can also lead to periodic behaviours, not shown in the Hopfield model, reproducing some biological aspects related to neuron circuits that control rhythmic activities, such as breathing, heart beating, walking.

For over two decades, several different generalizations of model (1.1), with and without delays, have been proposed, to include static neural networks, cellular networks, bidirectional associative

${ }^{1}$ Corresponding author. Fax:+351 21795 4288; Tel: +351 217904894. 
memory neural network systems, etc. Recently, the study of delayed differential equations (DDEs) modelling physiological or artificial neural networks has attracted a great attention among mathematicians and other scientists, and a significant number of publications has been produced. For this reason, here we avoid to give any description or realistic explanation of the models presented, since this can be easily found in the literature.

This paper deals with the analysis of the global stability for a very general class of CohenGrossberg-type autonomous neural network models with infinite distributed delay, of the form

$$
\dot{x}_{i}(t)=-a_{i}\left(x_{i}(t)\right)\left[b_{i}\left(x_{i}(t)\right)+\sum_{j=1}^{n} \sum_{p=1}^{P} f_{i j}^{(p)}\left(\int_{-\infty}^{0} g_{i j}^{(p)}\left(x_{j}(t+s)\right) d \eta_{i j}^{(p)}(s)\right)\right], i=1, \ldots, n,
$$

which includes all the particular models mentioned above. The results here extend the previous work in [6] and [22], where the case of bounded distributed delays was studied. See also [4], for the attractivity of equilibrium points to multi-species Lotka-Volterra systems with unbounded delay. We recall that systems with an infinite time-delay or "infinite memory" have been considered in population dynamics since the works of Volterra.

In fact, the Cohen-Grossberg model (1.3) will be treated here as a particular case of a much more general family of DDEs of the form

$$
\dot{x}_{i}(t)=-\rho_{i}\left(t, x_{t}\right)\left[b_{i}\left(x_{i}(t)\right)+f_{i}\left(x_{t}\right)\right], \quad i=1, \ldots, n,
$$

where $\rho_{i}, b_{i}, f_{i}$ are continuous real functions, $\rho_{i}$ are positive, and $x_{t}$ is defined by $x_{t}(s)=x(t+s)$ for $s \leq 0$.

For DDEs with infinite delay, the choice of an admissible Banach phase space (usually called fading memory space) should be made with special care, in order to obtain standard results of well-posedness of the initial value problem, existence, uniqueness and continuation of solutions, and precompactness of bounded positive orbits. Here, this task is facilitated since we shall always assume that the initial conditions are bounded on $(-\infty, 0]$. This is the usual setting in the literature on neural network systems with unbounded delay, and the reason why in most of the papers an explicit choice of the phase space is not even mentioned.

Many authors have investigated and analyzed several features of delayed differential equations representing neural networks, and it is impossible to mention all the significant works in the area. We however would like to refer to $[1,10,21,23,27,29]$ for their work on local stability and Hopf bifurcations, and $[1,2,9,19,24,25,26]$ for several criteria to ensure existence, global asymptotic stability, and global exponential stability of an equilibrium point.

Besides the above cited works, there is an extensive literature dealing with global stability of neural network models with delays. We emphasize however that the usual approach to study the global asymptotic stability of an equilibrium of a system relies on the Lyapunov functional technique. In general, constructing a Lyapunov functional for a concrete n-dimensional DDE is rather complex, and frequently a new Lyapunov functional for each model under consideration is required. In contrast with the usual method, our techniques (also in $[5,6]$ ) do not involve Lyapunov functionals, and apply to general systems. Moreover, most of the works on delayed neural networks consider only the case of a finite number of discrete delays, whereas we here treat the case of unbounded distributed delays.

The paper is organized as follows: In Section 2, we briefly present adequate Banach phase spaces for DDEs with infinite delay written in abstract form as $\dot{x}(t)=f\left(t, x_{t}\right)$, and establish a general condition for the boundedness of solutions and existence and uniform stability of the zero solution. In Section 3, we present the main results of the paper, on the existence, global asymptotic, and global exponential stability of an equilibrium point for the general class of DDEs with infinite delay (1.4), which includes most of the autonomous models of neural network systems. In Section 4, these results are applied to establish criteria for the global asymptotic, and the global exponential stability of equilibria for neuron network models (1.3). Section 5 is dedicated to applications of these criteria to concrete models. Throughout this section, a comparison of results with the literature is given, showing the advantage of our method when applied to several different models, such as cellular networks or bidirectional associative memory neural models. A short section with conclusions ends the paper. 


\section{Uniform stability}

Consider the following space, often referred to as "fading memory" space [11],

$$
U C_{g}=\left\{\phi \in C\left((-\infty, 0] ; \mathbb{R}^{n}\right): \sup _{s \leq 0} \frac{|\phi(s)|}{g(s)}<\infty, \frac{\phi(s)}{g(s)} \text { is uniformly continuous on }(-\infty, 0]\right\}
$$

where $g:(-\infty, 0] \rightarrow[1,+\infty)$ is a given function satisfying:

(g1) $g$ is a non-increasing continuous function and $g(0)=1$;

(g2) $\lim _{u \rightarrow 0^{-}} \frac{g(s+u)}{g(s)}=1$ uniformly on $(-\infty, 0]$;

(g3) $g(s) \rightarrow \infty$ as $s \rightarrow-\infty$.

For example, the function $g(s)=e^{-\alpha s}$ with $\alpha>0$ satisfies (g1)-(g3). The space $U C_{g}$ with the norm

$$
\|\phi\|_{g}=\sup _{s \leq 0} \frac{|\phi(s)|}{g(s)}
$$

where $|\cdot|$ is a fixed norm in $\mathbb{R}^{n}$, is a Banach space.

Consider also the space $B C=B C\left((-\infty, 0] ; \mathbb{R}^{n}\right)$ of bounded and continuous functions $\phi:(-\infty, 0] \rightarrow$ $\mathbb{R}^{n}$, and let $\|\cdot\|_{\infty}$ denote the supremum norm, $\|\phi\|_{\infty}=\sup _{s \leq 0}|\phi(s)|$. It is clear that $B C \subseteq U C_{g}$, with $\|\phi\|_{g} \leq\|\phi\|_{\infty}$ for $\phi \in B C$. When $B C$ is considered as a subspace of $U C_{g}$, we often write $B C_{g}$.

For an open set $D \subseteq U C_{g}$ and $f:[0, \infty) \times D \rightarrow \mathbb{R}^{n}$ continuous, consider the functional differential equation (FDE)

$$
\dot{x}(t)=f\left(t, x_{t}\right), \quad t \geq 0,
$$

where, as usual, $x_{t}$ denotes the function $x_{t}:(-\infty, 0] \rightarrow \mathbb{R}^{n}$ defined by $x_{t}(s)=x(t+s)$ for $s \leq 0$. Since $g$ satisfies (g1)-(g3), the phase space $U C_{g}$ is an admissible Banach space for $(2.1)$ in the sense of [11] and therefore standard existence, uniqueness and continuation type results are valid [13]. We always assume that $f$ is regular enough in order to have uniqueness of solution for the initial value problem. The solution of $(2.1)$ with initial condition $x_{t_{0}}=\varphi$ is denoted by $x\left(t, t_{0}, \varphi\right)$.

In view of our applications to neural network systems, we restrict our attention to initial bounded conditions, i.e.,

$$
x_{t_{0}}=\varphi, \quad \text { with } \varphi \in B C,
$$

for some $t_{0} \geq 0$. From [13], if $f$ takes closed bounded subsets of its domain into bounded sets of $\mathbb{R}^{n}$, then the solution of (2.1)-(2.2) is extensible to intervals $[0, a]$ whenever it is bounded.

In [5], a result on the boundedness of solutions for a general FDE (2.1) with finite delay was established, when the norm $|x|=\max \left\{\left|x_{i}\right|: i=1, \ldots, n\right\}, x=\left(x_{1}, \ldots, n\right) \in \mathbb{R}^{n}$, is chosen in $\mathbb{R}^{n}$. Here, a generalization of such result is given, with the same norm in $\mathbb{R}^{n}$, but for the case of unbounded delays.

Lemma 2.1. Consider equation (2.1) in $U C_{g}$, and suppose that $f$ transforms closed bounded sets of $[0, \infty) \times D$ into bounded sets of $\mathbb{R}^{n}$. Assume also that

(H1) for all $t \geq 0$ and $\varphi \in U C_{g}$ such that $\frac{|\varphi(s)|}{g(s)}<|\varphi(0)|$, for $s \in(-\infty, 0)$, then $\varphi_{i}(0) f_{i}(t, \varphi)<0$ for some $i \in\{1, \ldots, n\}$ such that $|\varphi(0)|=\left|\varphi_{i}(0)\right|$.

Then, the solutions $x(t)=x(t, 0, \varphi), \varphi \in U C_{g}$, of (2.1) are defined on $[0, \infty)$ and satisfy $|x(t, 0, \varphi)| \leq$ $\|\varphi\|_{g}$ for $t \geq 0$. 
Proof. From [13], it follows that a solution with an initial condition $x_{0}=\varphi \in U C_{g}$ is defined for $t \geq 0$ if it is bounded on every interval $[0, a](a>0)$. We now prove that solutions $x(t)$ defined on $[0, a]$ satisfy $|x(t)| \leq\left\|x_{0}\right\|_{g}$ for $0 \leq t \leq a$. The proof is similar to the one of Lemma 3.2 of [5] (see also Theorem 3.1 of [4]). For convenience of the reader, we include it here.

Let $x(t)=x(t, 0, \varphi)$ be a solution of $(2.1)$ on $[0, a]$ for some $a>0$, with $\|\varphi\|_{g}=k$. Suppose that there is $t_{1}>0$ such that $\left|x\left(t_{1}\right)\right|>k$ and define

$$
T=\min \left\{t \in\left[0, t_{1}\right]: \max _{s \in\left[0, t_{1}\right]}|x(s)|=|x(t)|\right\} .
$$

We have $|x(T)|>k$ and

$$
\frac{\left|x_{T}(s)\right|}{g(s)}=\frac{|x(T+s)|}{g(s)} \leq \frac{|x(T+s)|}{g(T+s)} \leq k<|x(T)| \quad \text { for } \quad s \leq-T,
$$

and

$$
\frac{\left|x_{T}(s)\right|}{g(s)} \leq\left|x_{T}(s)\right|=|x(T+s)|<|x(T)| \quad \text { for } \quad s \in[-T, 0) .
$$

Hence $\left|x_{T}(s)\right| / g(s)<|x(T)|$ for $s \in(-\infty, 0)$. By (H1) there is $i \in\{1, \ldots, n\}$ such that $\left|x_{i}(T)\right|=$ $|x(T)|$ and $x_{i}(T) f_{i}\left(T, x_{T}\right)<0$. Suppose that $x_{i}(T)>0$ (the situation $x_{i}(T)<0$ is analogous). Since $x_{i}(t) \leq|x(t)|<x_{i}(T)$ for $t \in[0, T)$, then $\dot{x}_{i}(T) \geq 0$. On the other hand we have $\dot{x}_{i}(T)=f_{i}\left(T, x_{T}\right)<$ 0 , a contradiction. This proves that $|x(t, 0, \varphi)| \leq\|\varphi\|_{g}$ for all $t \geq 0$, whenever $x(t, 0, \varphi)$ is defined.

In order to obtain boundedness of solutions and uniform stability of the zero solution in $B C$, rather than (H1) we can impose a less restrictive hypothesis, as stated in the next lemma. The proof is similar to the one above, and therefore omitted.

Lemma 2.2. Consider equation (2.1) in $U C_{g}$, and suppose that $f$ transforms closed bounded sets of $[0, \infty) \times D$ into bounded sets of $\mathbb{R}^{n}$. Assume also that

(H2) for all $t \geq 0$ and $\varphi \in B C$ such that $|\varphi(s)|<|\varphi(0)|$, for $s \in(-\infty, 0)$, then $\varphi_{i}(0) f_{i}(t, \varphi)<0$ for some $i \in\{1, \ldots, n\}$ such that $|\varphi(0)|=\left|\varphi_{i}(0)\right|$.

Then, all solutions of (2.1) with initial conditions in BC are defined and bounded on $[0, \infty)$. Moreover, if $x(t)=x(t, 0, \varphi), \varphi \in B C$, is a solution of (2.1) then $|x(t, 0, \varphi)| \leq\|\varphi\|_{\infty}$ for all $t \geq 0$.

\section{Main results}

In this section, we study the global asymptotic and the global exponential stability of an equilibrium point for a family of FDEs with infinite delays given in abstract form as

$$
\dot{x}_{i}(t)=-\rho_{i}\left(t, x_{t}\right)\left[b_{i}\left(x_{i}(t)\right)+f_{i}\left(x_{t}\right)\right], \quad i=1, \ldots, n, \quad t \geq 0
$$

where $\rho_{i}:[0, \infty) \times U C_{g} \rightarrow(0, \infty), b_{i}: \mathbb{R} \rightarrow \mathbb{R}$ and $f_{i}: U C_{g} \rightarrow \mathbb{R}$ are continuous, $i=1, \ldots, n$.

This general class of FDEs includes most of the (autonomous) neural network models with infinite delay present in the literature, as shown in Sections 4 and 5. As mentioned before, for neural network models with unbounded delays, the initial conditions are always assumed to be bounded. Therefore, throughout the paper we take $B C$ as the set of admissible initial conditions, and only consider solutions of general models (3.1) with initial conditions (2.2).

As usual, for a vector $a=\left(a_{1}, \ldots, a_{n}\right) \in \mathbb{R}^{n}$, we also write $a$ to denote the constant function $\varphi(s) \equiv a$ in $B C$, or $U C_{g}$.

Definition 3.1. If $x^{*} \in \mathbb{R}^{n}$ is an equilibrium of (3.1), $x^{*}$ is said to be globally asymptotically stable (in the set of admissible solutions) if it globally attractive in $\mathbb{R}^{n}$, i.e., $x(t) \rightarrow x^{*}$ as $t \rightarrow \infty$, for all solutions $x(t)$ with initial conditions in $B C_{g}$, and is stable in $U C_{g}$; and $x^{*}$ is said to be globally exponentially stable if there are positive constants $\varepsilon, M$ such that

$$
\left|x(t, 0, \varphi)-x^{*}\right| \leq M e^{-\varepsilon t}\left\|\varphi-x^{*}\right\|_{\infty}, \quad \text { for all } t \geq 0, \varphi \in B C .
$$


It should be mentioned that the above definition of global exponential stability of an equilibrium $x^{*}$ is the usual in the literature on neural networks with unbounded delay, but it does not even imply the stability of $x^{*}$ in the phase space $U C_{g}$, i.e., relative to the norm $\|\cdot\|_{g}$.

In the sequel, for (3.1) the following hypotheses will be considered:

(A1) For any $M>0, \sup \left\{\rho_{i}(t, \phi): \phi \in B C,\|\phi\|_{\infty} \leq M, t \geq 0\right\}<\infty$ and $r_{i}(t):=\inf \left\{\rho_{i}(t, \phi): \phi \in\right.$ $\left.B C,\|\phi\|_{\infty} \leq M\right\}$ satisfies $\int_{0}^{\infty} r_{i}(t) d t=\infty, i \in\{1, \ldots, n\}$;

(A2) for each $i \in\{1, \ldots, n\}$, there is $\beta_{i}>0$ such that

$$
\left(b_{i}(u)-b_{i}(v)\right) /(u-v) \geq \beta_{i}, \quad \forall u, v \in \mathbb{R}, u \neq v
$$

(A3) $f_{i}: U C_{g} \rightarrow \mathbb{R}$ is a Lipschitz function with Lipschitz constant $l_{i}, i \in\{1, \ldots, n\}$;

(A4) $\beta_{i}>l_{i}$ for all $i \in\{1, \ldots, n\}$.

Lemma 3.1. Assume (A2), (A3) and (A4). Then system (3.1) has a unique equilibrium point $x^{*}=\left(x_{1}^{*}, \ldots, x_{n}^{*}\right) \in \mathbb{R}^{n}$.

Proof. Define the continuous function $H: \mathbb{R}^{n} \rightarrow \mathbb{R}^{n}, H(x)=\left(b_{1}\left(x_{1}\right)+f_{1}(x), \ldots, b_{n}\left(x_{n}\right)+f_{n}(x)\right)$ for $x=\left(x_{1}, \ldots, x_{n}\right)$. Under the assumptions, reasoning as Lemma 2.4 in [22] one proves that $H$ is injective and that $|H(x)| \rightarrow \infty$ as $|x| \rightarrow \infty$. For more details, cf. [22]. Making use of a lemma in [8], we conclude that $H$ is a homeomorphism of $\mathbb{R}^{n}$, and therefore there is a unique $x^{*} \in \mathbb{R}^{n}$ such that $H\left(x^{*}\right)=0$.

Now, we state our main result on the global asymptotic stability of the equilibrium $x^{*}$ of (3.1).

Theorem 3.2. Assume (A1)-(A4). Then system (3.1) has a unique equilibrium point which is globally asymptotically stable.

Proof. From Lemma 3.1, system (3.1) has a unique equilibrium point $x^{*}=\left(x_{1}^{*}, \ldots, x_{n}^{*}\right) \in \mathbb{R}^{n}$. Translating $x^{*}$ to the origin by the change $\bar{x}(t)=x(t)-x^{*},(3.1)$ becomes

$$
\left.\dot{\bar{x}}_{i}(t)=-\bar{\rho}_{i}\left(t, \bar{x}_{t}\right)\right)\left[\bar{b}_{i}\left(\bar{x}_{i}(t)\right)+\bar{f}_{i}\left(\bar{x}_{t}\right)\right], \quad i=1, \ldots, n, \quad t \geq 0,
$$

where $\bar{\rho}_{i}(t, \varphi)=\rho_{i}\left(t, \varphi+x^{*}\right), \bar{b}_{i}(u)=b_{i}\left(u+x_{i}^{*}\right)$ and $\bar{f}_{i}(\varphi)=f_{i}\left(x^{*}+\varphi\right)$, with zero as the unique equilibrium point, i.e. $\bar{b}_{i}(0)+\bar{f}_{i}(0)=0$ for $i=1, \ldots, n$. Clearly $\rho_{i}, b_{i}$ and $f_{i}$ satisfy (A1)-(A4) if and only if $\bar{\rho}_{i}, \bar{b}_{i}$ and $\bar{f}_{i}$ satisfy (A1)-(A4). Hence, we consider (3.2), where, for simplicity, we drop the bars.

Let $\varphi \in B C_{g}$ be such that $\|\varphi\|_{g}=|\varphi(0)|>0$ and consider $i \in\{1, \ldots, n\}$ such that $\left|\varphi_{i}(0)\right|=\|\varphi\|_{g}$. If $\varphi_{i}(0)>0$ (the situation $\varphi_{i}(0)<0$ is analogous), then $\|\varphi\|_{g}=\varphi_{i}(0)$ and from the hypotheses we conclude that

$$
\begin{aligned}
b_{i}\left(\varphi_{i}(0)\right)+f_{i}(\varphi) & =\left[b_{i}\left(\varphi_{i}(0)\right)-b_{i}(0)\right]+\left[f_{i}(\varphi)-f_{i}(0)\right] \\
& \geq\left(\beta_{i}-l_{i}\right)\|\varphi\|_{g}>0
\end{aligned}
$$

In particular, (H1) holds and from Lemma 2.1 we deduce that all solutions are defined and bounded on $[0, \infty)$, and that $x=0$ is uniformly stable. It remains to prove that zero is globally attractive.

For $x(t)=\left(x_{i}(t)\right)_{i=1}^{n}$ a solution of (3.2), define the limits

$$
-v_{i}=\liminf _{t \rightarrow \infty} x_{i}(t), \quad u_{i}=\limsup _{t \rightarrow \infty} x_{i}(t), \quad i=1, \ldots, n,
$$

and

$$
v=\max _{i}\left\{v_{i}\right\}, \quad u=\max _{i}\left\{u_{i}\right\}
$$


Note that $u, v \in \mathbb{R}$ and $-v \leq u$. It is sufficient to prove that $\max (u, v)=0$. Assume e.g. that $|v| \leq u$, so that $\max (u, v)=u$. (The situation is analogous for $|u| \leq v$.)

Let $i \in\{1, \ldots, n\}$ be such that $u_{i}=u$. Now we denote $\overline{h_{i}}(\varphi):=-\left[b_{i}\left(\varphi_{i}(0)\right)+f_{i}(\varphi)\right]$, for $\varphi \in B C_{g}$, and prove that there is a sequence $\left(t_{k}\right)_{k \in \mathbb{N}}$ such that

$$
t_{k} \nearrow \infty, \quad x_{i}\left(t_{k}\right) \rightarrow u, \quad \text { and } \quad h_{i}\left(x_{t_{k}}\right) \rightarrow 0, \text { as } k \rightarrow \infty
$$

Case 1. Assume that $x_{i}(t)$ is eventually monotone. In this case, $\lim _{t \rightarrow \infty} x_{i}(t)=u$ and for $t$ large, either $\dot{x}_{i}(t) \leq 0$ or $\dot{x}_{i}(t) \geq 0$. Assume e.g. that $\dot{x}_{i}(t) \leq 0$ for $t$ large (the situation $\dot{x}_{i}(t) \geq 0$ is analogous). Then $h_{i}\left(x_{t}\right) \leq 0$ for $t$ large, hence

$$
\limsup _{t \rightarrow \infty} h_{i}\left(x_{t}\right):=c \leq 0 .
$$

For $M=\sup _{t \in \mathbb{R}}|x(t)|$, consider $r_{i}(t)=\inf \left\{\rho_{i}(t, \phi):\|\phi\|_{\infty} \leq M\right\}$. If $c<0$, then there is $t_{0}>0$ such that $h_{i}\left(x_{t}\right)<c / 2$ for $t \geq t_{0}$, implying that

$$
x_{i}(t) \leq x_{i}\left(t_{0}\right)+\frac{c}{2} \int_{t_{0}}^{t} r_{i}(s) d s .
$$

From (A1) and the above inequality, we obtain $x_{i}(t) \rightarrow-\infty$ as $t \rightarrow \infty$, which is not possible. Thus $c=0$, which proves (3.4).

Case 2. Assume that $x_{i}(t)$ is not eventually monotone. In this case there is a sequence $\left(t_{k}\right)_{k \in \mathbb{N}}$ such that $t_{k} \nearrow \infty, \dot{x}_{i}\left(t_{k}\right)=0$ and $x_{i}\left(t_{k}\right) \rightarrow u$, as $k \rightarrow \infty$. Then $h_{i}\left(x_{t_{k}}\right)=0$ for all $k \in \mathbb{N}$, and (3.4) holds.

Next, we show that $u=0$, hence $v=0$ as well.

Since $x(t)$ is bounded on $[0, \infty)$, then there is $L>0$ such that $\left\|x_{t}\right\|_{g}<L$ for all $t \geq 0$. Hence, from (A1), (A3) we conclude that there is $K>0$ such that $\left|\dot{x}_{j}(t)\right|=\left|\rho_{j}\left(t, x_{t}\right)\left[b_{j}\left(x_{j}(t)\right)+f_{j}\left(x_{t}\right)\right]\right|<K$, for all $t \geq 0$ and $j \in\{1, \ldots, n\}$. Together with the fact that the initial condition $x_{0}$ is bounded on $(-\infty, 0]$ and Theorem 3.1 in [11], this estimate implies that $\left\{x_{t}: t \geq 0\right\}$ is precompact in $U C_{g}$. Thus, for a subsequence of $\left(x_{t_{k}}\right)_{k}$, still denoted by $\left(x_{t_{k}}\right)_{k}$, there is $\phi \in U C_{g}$ such that $x_{t_{k}} \rightarrow \phi$ in $U C_{g}$ as $k \rightarrow \infty$. On the other hand, let $\varepsilon>0$ be fixed. There is $T=T(\varepsilon)>0$ such that $|x(t)|<u_{\varepsilon}:=u+\varepsilon$ for $t \geq T$, therefore for any $s \leq 0$ we obtain $|\phi(s)| / g(s) \leq\left\|x_{t_{k}}-\phi\right\|_{g}+\left|x\left(t_{k}+s\right)\right| / g(s) \leq\left\|x_{t_{k}}-\phi\right\|_{g}+u_{\varepsilon}$ for $k$ large, hence $\|\phi\|_{g} \leq u_{2 \varepsilon}$. Since $\varepsilon>0$ is arbitrary, we conclude that $\|\phi\|_{g} \leq u$. From (3.4), we get $\phi_{i}(0)=u$ and $h_{i}(\phi)=0$. Clearly $\|\phi\|_{g}=\left|\phi_{i}(0)\right|=u$. Now, if $u>0$, arguing as in (3.3) we conclude that $h_{i}(\phi)<0$, which is not possible. As a consequence, $u=0$ and the theorem is proven.

Remark 3.1. As a particular case, we can consider the subclass of FDEs (3.1) where $\rho_{i}(t, \phi)=$ $r_{i}(t) a_{i}\left(\phi_{i}(0)\right)$, with $r_{i}:[0, \infty) \rightarrow(0, \infty)$ and $a_{i}: \mathbb{R} \rightarrow(0, \infty)$ continuous, so that $(3.1)$ becomes

$$
\dot{x}_{i}(t)=-r_{i}(t) a_{i}\left(x_{i}(t)\right)\left[b_{i}\left(x_{i}(t)\right)+f_{i}\left(x_{t}\right)\right], \quad i=1, \ldots, n, \quad t \geq 0 .
$$

In this situation, hypothesis (A1) is written in a simpler form as follows:

(A1') $r_{i}(t)$ is uniformly bounded on $[0, \infty)$ and $\int_{0}^{\infty} r_{i}(t) d t=\infty, i \in\{1, \ldots, n\}$.

Remark 3.2. In the proof of Therorem 3.2, it was crucial to have a result on precompactness for positive orbits of (3.1). (In fact, the same argument shows that $\left\|x_{t}-x^{*}\right\|_{g} \rightarrow 0$ as $t \rightarrow \infty$ for any solution $x(t)$ with initial data in $B C$, hence $x^{*}$ is globally attractive in $U C_{g}$.) From [11], positive orbits of solutions $x(t)$ which are bounded and uniformly continuous on $[0, \infty)$ are always precompact in $U C_{g}$, provided that $|x(s)| / g(s) \rightarrow 0$ as $s \rightarrow-\infty$. Clearly this latter condition always holds if we only consider initial conditions $x_{0}=\phi$ with $\phi \in B C$. On the other hand, Theorem 3.2 in [11] asserts that, if $U C_{g}$ is a "strong fading memory" space, then the positive orbit $\left\{x_{t}: t \geq 0\right\}$ of a solution $x(t)$ which is bounded and uniformly continuous on $[0, \infty)$ is precompact. Therefore, for this situation we can relax our initial constraint, and take the full space $U C_{g}$ as the set of admissible initial conditions.

We now address the global exponential stability of systems (3.1). For that, we consider the space $U C_{g}$ with $g(s)=e^{-\alpha s}, s \in(-\infty, 0]$, for some $\alpha>0$. Recall that, with such choice of $g, U C_{g}$ is a 
strong fading memory space (see Theorem 3.2 of [11]). In the literature, we find examples of neural networks given by FDEs with infinite delays, for which $U C_{g}$ with $g$ of the above form $g(s)=e^{-\alpha s}$ (for some $\alpha>0$ to be fixed) may be taken as the phase space, although that is not always explicit (see e.g. in [23]). In fact, as already noticed, most papers dealing with neural networks with unbounded delay do not provide an explicit phase space. In the sequel, as before we always consider bounded initial conditions $x_{0}=\phi \in B C$.

Theorem 3.3. Consider system (3.1) in $U C_{g}$, for $g(s)=e^{-\alpha s}, s \in(-\infty, 0]$, for some $\alpha>0$. Assume (A2), (A3), (A4), and

$\left(\mathbf{A} 1^{*}\right) \underline{\rho}:=\inf \left\{\rho_{i}(t, \varphi): t \geq 0, \varphi \in B C_{g}, 1 \leq i \leq n\right\}>0$.

Then the unique equilibrium $x^{*}$ of (3.1) is globally exponentially stable.

Proof. As in the above proof, after a translation, we may assume that the equilibrium point is zero, i.e., $b_{i}(0)+f_{i}(0)=0$ for $i=1, \ldots, n$.

Since $\beta_{i}>l_{i}$ and $\underline{\rho}>0$, choose $\varepsilon \in(0, \alpha)$ such that $\varepsilon-\rho\left(\beta_{i}-l_{i}\right)<0$ for all $i=1, \ldots, n$.

Let $x(t)=x(t, 0, \bar{\varphi})$ be a solution of (3.1). The change of variables $z(t)=e^{\varepsilon t} x(t)$ transforms $(3.1)$ into

$$
\dot{z}(t)=F_{i}\left(t, z_{t}\right), \quad t \geq 0, \quad i=1, \ldots, n,
$$

where

$$
F_{i}(t, \phi)=\varepsilon \phi_{i}(0)-\rho_{i}\left(t, e^{-\varepsilon(t+\cdot)} \phi\right) e^{\varepsilon t}\left[b_{i}\left(e^{-\varepsilon t} \phi_{i}(0)\right)+f_{i}\left(e^{-\varepsilon(t+\cdot)} \phi\right)\right] .
$$

Let $t \geq 0$ and $\phi \in B C$ such that $|\phi(s)|<|\phi(0)|$, for $s \in(-\infty, 0)$, and consider $i \in\{1, \ldots, n\}$ such that $\left|\phi_{i}(0)\right|=|\phi(0)|$.

If $\phi_{i}(0)>0$ (the situation $\phi_{i}(0)<0$ is analogous), then our hypotheses imply that

$$
\begin{aligned}
F_{i}(t, \phi) & =\varepsilon \phi_{i}(0)-\rho_{i}\left(t, e^{-\varepsilon(t+\cdot)} \phi\right) e^{\varepsilon t}\left[b_{i}\left(e^{-\varepsilon t} \phi_{i}(0)\right)-b_{i}(0)+f_{i}\left(e^{-\varepsilon(t+\cdot)} \phi\right)-f_{i}(0)\right] \\
& \leq \varepsilon \phi_{i}(0)-\rho_{i}\left(t, e^{-\varepsilon(t+\cdot)} \phi\right) e^{\varepsilon t}\left[\beta_{i} e^{-\varepsilon t} \phi_{i}(0)-l_{i}\left\|e^{-\varepsilon(t+\cdot)} \phi\right\|_{g}\right] \\
& \leq \varepsilon \phi_{i}(0)-\underline{\rho} e^{\varepsilon t}\left[\beta_{i} e^{-\varepsilon t} \phi_{i}(0)-l_{i} \sup _{s \leq 0} \frac{e^{-\varepsilon t} e^{-\varepsilon s}|\phi(s)|}{e^{-\alpha s}}\right] \\
& \leq \varepsilon \phi_{i}(0)-\underline{\rho}\left[\beta_{i} \phi_{i}(0)-l_{i} \sup _{s \leq 0} e^{(\alpha-\varepsilon) s}|\phi(s)|\right] .
\end{aligned}
$$

Since $\alpha-\varepsilon>0$, we have

$$
F_{i}(t, \phi) \leq \phi_{i}(0)\left[\varepsilon-\underline{\rho}\left(\beta_{i}-l_{i}\right)\right]<0,
$$

and (H2) holds for $F=\left(F_{1}, \ldots, F_{n}\right)$. From Lemma 2.2, the solution $z(t)$ is defined on $[0, \infty)$ and satisfies $|z(t)| \leq \sup _{s \leq 0}|z(s)|$ for $t \geq 0$. Thus we obtain

$$
|x(t, 0, \varphi)|=\left|e^{-\varepsilon t} z\left(t, 0, e^{\varepsilon \cdot} \varphi\right)\right| \leq e^{-\varepsilon t} \sup _{s \leq 0}|\varphi(s)|, \quad t \geq 0, \varphi \in B C .
$$

We remark that the above result extends to the infinite delay case a previous criterion presented in [6] for FDEs with finite delays.

Clearly, the above theorems can be generalized for non-autonomous models as follows: 
Theorem 3.4. Consider

$$
\dot{x}_{i}(t)=-\rho_{i}\left(t, x_{t}\right)\left[b_{i}\left(x_{i}(t)\right)+f_{i}\left(t, x_{t}\right)\right], \quad i=1, \ldots, n, \quad t \geq 0,
$$

with $\rho_{i}, b_{i}$ as in (3.1) and $f_{i}:[0, \infty) \times U C_{g} \rightarrow \mathbb{R}$ continuous, and assume that there is an equilibrium point $x^{*}=\left(x_{1}^{*}, \ldots, x_{n}^{*}\right) \in \mathbb{R}^{n}$. Then, the statements of Theorems 3.2 and 3.3 on the stability of $x^{*}$ are valid with (A3) replaced by the condition of $f_{i}$ being uniformly $l_{i}$-Lipschitzian with respect to the variable $\varphi \in U C_{g}$, i.e., for $i=1, \ldots, n$,

$\left(\mathbf{A} 3^{*}\right)\left|f_{i}(t, \varphi)-f_{i}(t, \psi)\right| \leq l_{i}\|\varphi-\psi\|_{g}$, for $t \geq 0$ and $\varphi, \psi \in U C_{g}$.

\section{Cohen-Grossberg neural networks}

In this section, we apply the previous results to the following generalized Cohen-Grossberg neural network model with infinite distributed delays:

$$
\dot{x}_{i}(t)=-a_{i}\left(x_{i}(t)\right)\left[b_{i}\left(x_{i}(t)\right)+\sum_{j=1}^{n} \sum_{p=1}^{P} f_{i j}^{(p)}\left(\int_{-\infty}^{0} g_{i j}^{(p)}\left(x_{j}(t+s)\right) d \eta_{i j}^{(p)}(s)\right)\right], \quad i=1, \ldots, n
$$

where $a_{i}: \mathbb{R} \rightarrow(0, \infty), b_{i}: \mathbb{R} \rightarrow \mathbb{R}$ and $f_{i j}^{(p)}, g_{i j}^{(p)}: \mathbb{R} \rightarrow \mathbb{R}$ are continuous functions, and $\eta_{i j}^{(p)}:$ $(-\infty, 0] \rightarrow \mathbb{R}$ are non-decreasing bounded functions, normalized so that $\eta_{i j}^{(p)}(0)-\eta_{i j}^{(p)}(-\infty)=1$, for all $i, j \in\{1, \ldots, n\}, p \in\{1, \ldots, P\}$. We further assume that the functions $b_{i}$ satisfy (A2) and that $f_{i j}^{(p)}, g_{i j}^{(p)}$ are Lipschitzian with Lipschitz constants $\mu_{i j}^{(p)}, \sigma_{i j}^{(p)}$, respectively. For $(4.1), B C$ is taken as the set of initial conditions, which guarantees that solutions are extensible to $[0, \infty)$. Model (4.1) is particularly relevant in terms of applications, as we shall illustrate extensively in the next section with several examples.

Define the square real matrices,

$$
B=\operatorname{diag}\left(\beta_{1}, \ldots, \beta_{n}\right), \quad L=\left[l_{i j}\right] \quad \text { and } \quad N=B-L,
$$

where $\beta_{1}, \ldots, \beta_{n}$ are as in (A2) and

$$
l_{i j}=\sum_{p=1}^{P} \mu_{i j}^{(p)} \sigma_{i j}^{(p)}, \quad i, j=1, \ldots, n .
$$

We now prove an auxiliary result which is a generalization of a result in [12].

Lemma 4.1. Consider $\eta_{i}:(-\infty, 0] \rightarrow \mathbb{R}, i=1, \ldots, m$, non-decreasing and bounded functions, and $\alpha>0$ such that

$$
\int_{-\infty}^{0} d \eta_{i}(s)<\alpha, \quad i=1, \ldots, m
$$

Then, there is a continuous function $g:(-\infty, 0] \rightarrow[1,+\infty)$ satisfying $(\mathbf{g} 1)$, (g2) and (g3), and such that

$$
\int_{-\infty}^{0} g(s) d \eta_{i}(s)<\alpha, \quad i=1, \ldots, m
$$

Proof. We use arguments similar to the ones in [12]. First, define

$$
\alpha_{i}:=\eta_{i}(0)-\eta(-\infty)=\int_{-\infty}^{0} d \eta_{i}(s)<\alpha, \quad i=1, \ldots, m .
$$

For each $n \in \mathbb{N}$ and $i \in\{1, \ldots, m\}$, let $\varepsilon_{i, n}=\left(\alpha-\alpha_{i}\right) /\left[2^{n+1}(n+1)\right]$. Since $\eta_{i}$ is non-decreasing and bounded, there is a sequence $\left(r_{n}\right)_{n \in \mathbb{N}}$ of positive real numbers (independent of $i$ ) such that $r_{n+1} \geq r_{n}+1$ and

$$
\int_{-\infty}^{-r_{n}} d \eta_{i}(s)<\varepsilon_{i, n}, \quad i=1, \ldots, m, n \in \mathbb{N} .
$$

Now, define $g:(-\infty, 0] \rightarrow[1,+\infty)$ as follows: 
(i) $g(s)=1$ on $\left[-r_{1}, 0\right]$;

(ii) $g\left(-r_{n}\right)=n, n \in \mathbb{N}$;

(iii) $g$ is continuous and piecewise linear (linear on intervals $\left[-r_{n+1},-r_{n}\right]$ ).

From (i) and (4.3), we have

$$
\int_{-r_{1}}^{0} g(s) d \eta_{i}(s)<\frac{\alpha+\alpha_{i}}{2} .
$$

Hence, for each $i=1, \ldots, m$, we have

$$
\begin{aligned}
\int_{-\infty}^{0} g(s) d \eta_{i}(s) & =\int_{-r_{1}}^{0} g(s) d \eta_{i}(s)+\sum_{n=1}^{\infty} \int_{-r_{n+1}}^{-r_{n}} g(s) d \eta_{i}(s) \\
& <\frac{\alpha+\alpha_{i}}{2}+\sum_{n=1}^{\infty} g\left(-r_{n+1}\right) \int_{-r_{n+1}}^{-r_{n}} d \eta_{i}(s) \\
& \leq \frac{\alpha+\alpha_{i}}{2}+\sum_{n=1}^{\infty}(n+1) \varepsilon_{i, n}=\frac{\alpha+\alpha_{i}}{2}+\sum_{n=1}^{\infty} \frac{\alpha-\alpha_{i}}{2^{n+1}}=\alpha .
\end{aligned}
$$

The stability of (4.1) is strongly related to the algebraic properties of the associated matrix $N$ defined in (4.2). We recall here the definition of a non-singular M-matrix. For further properties of M-matrices, we refer the reader to Chapter 5 of [7].

Definition 4.1. If $D=\left[d_{i j}\right]$ is a square matrix with non-positive off-diagonal entries, i.e., $d_{i j} \leq 0$ for all $i \neq j$, we say that $D$ is a non-singular $M$-matrix if all the eigenvalues of $D$ have positive real part, or, equivalently, if all the principal minors of $D$ are positive.

Theorem 4.2. For system (4.1), assume (A2), that $f_{i j}^{(p)}, g_{i j}^{(p)}$ are Lipschitz functions with Lipschitz constants $\mu_{i j}^{(p)}, \sigma_{i j}^{(p)}$, respectively, and that $\eta_{i j}^{(p)}$ are non-decreasing bounded functions, normalized so that $\eta_{i j}^{(p)}(0)-\eta_{i j}^{(p)}(-\infty)=1$, for all $i, j \in\{1, \ldots, n\}, p \in\{1, \ldots, P\}$.

For $N$ defined in (4.2), if $N$ is a non-singular $M$-matrix, then there is a unique equilibrium point of (4.1), which is globally asymptotically stable.

Proof. If $N$ is a non-singular M-matrix, then (see [7]) there is $d=\left(d_{1}, \ldots, d_{n}\right)>0$ such that $N d>0$, i.e.,

$$
\beta_{i} d_{i}>\sum_{j=1}^{n} l_{i j} d_{j}, \quad i=1, \ldots, n,
$$

hence, there is $\delta>0$ such that

$$
\beta_{i} d_{i}>\sum_{j=1}^{n} l_{i j}(1+\delta) d_{j}, \quad i=1, \ldots, n .
$$

Since $\int_{-\infty}^{0} d \eta_{i j}^{(p)}(s)<1+\delta$ for $i, j=1, \ldots, n, p=1, \ldots, P$, from Lemma 4.1 we conclude that there is $g:(-\infty, 0] \rightarrow[1,+\infty)$ satisfying (g1)-(g3) such that

$$
\int_{-\infty}^{0} g(s) d \eta_{i j}^{(p)}(s)<1+\delta
$$

The change $y_{i}(t)=d_{i}^{-1} x_{i}(t)$ transforms (4.1) into the system

$$
\dot{y}_{i}(t)=-a_{i}\left(d_{i} y_{i}(t)\right) d_{i}^{-1}\left[b_{i}\left(d_{i} y_{i}(t)\right)+\sum_{j=1}^{n} \sum_{p=1}^{P} f_{i j}^{(p)}\left(\int_{-\infty}^{0} g_{i j}^{(p)}\left(d_{j} y_{j}(t+s)\right) d \eta_{i j}^{(p)}(s)\right)\right],
$$


for which we consider $U C_{g}$ as the phase space. For each $i \in\{1, \ldots, n\}$, define

$$
\bar{f}_{i}(\phi)=d_{i}^{-1} \sum_{j=1}^{n} \sum_{p=1}^{P} f_{i j}^{(p)}\left(\int_{-\infty}^{0} g_{i j}^{(p)}\left(d_{j} \phi_{j}(s)\right) d \eta_{i j}^{(p)}(s)\right), \quad \phi=\left(\phi_{1}, \ldots, \phi_{n}\right) \in U C_{g},
$$

and

$$
\bar{b}_{i}(u)=d_{i}^{-1} b_{i}\left(d_{i} u\right), \quad \bar{a}_{i}=a_{i}\left(d_{i} u\right), \quad u \in \mathbb{R} .
$$

System (4.5) is written as

$$
\dot{y}_{i}(t)=-\bar{a}_{i}\left(y_{i}(t)\right)\left[\bar{b}_{i}\left(y_{i}(t)\right)+\bar{f}_{i}\left(y_{t}\right)\right], \quad i=1, \ldots, n, t \geq 0 .
$$

For $\phi, \psi \in U C_{g}$ and $i=1, \ldots, n$, since $f_{i j}^{p}, g_{i j}^{p}$ are Lipschitz continuous and $\eta_{i j}^{(p)}$ are non-decreasing, we have

$$
\begin{aligned}
\left|\bar{f}_{i}(\phi)-\bar{f}_{i}(\psi)\right| & =d_{i}^{-1}\left|\sum_{j=1}^{n} \sum_{p=1}^{P}\left(f_{i j}^{(p)}\left(\int_{-\infty}^{0} g_{i j}^{(p)}\left(d_{j} \phi_{j}(s)\right) d \eta_{i j}^{(p)}(s)\right)-f_{i j}^{(p)}\left(\int_{-\infty}^{0} g_{i j}^{(p)}\left(d_{j} \psi_{j}(s)\right) d \eta_{i j}^{(p)}(s)\right)\right)\right| \\
& \leq d_{i}^{-1} \sum_{j=1}^{n} \sum_{p=1}^{P} \mu_{i j}^{(p)}\left|\int_{-\infty}^{0} g_{i j}^{(p)}\left(d_{j} \phi_{j}(s)\right)-g_{i j}^{(p)}\left(d_{j} \psi_{j}(s)\right) d \eta_{i j}^{(p)}(s)\right| \\
& \leq d_{i}^{-1} \sum_{j=1}^{n} d_{j} \sum_{p=1}^{P} \mu_{i j}^{(p)} \sigma_{i j}^{(p)} \int_{-\infty}^{0} g(s) \frac{\left|\left(\phi_{j}-\psi_{j}\right)(s)\right|}{g(s)} d \eta_{i j}^{(p)}(s) \\
& \leq\left(d_{i}^{-1} \sum_{j=1}^{n} d_{j} \sum_{p=1}^{P} \mu_{i j}^{(p)} \sigma_{i j}^{(p)} \int_{-\infty}^{0} g(s) d \eta_{i j}^{(p)}(s)\right)\|\phi-\psi\|_{g} \\
& \leq\left(d_{i}^{-1} \sum_{j=1}^{n} l_{i j}(1+\delta) d_{j}\right)\|\phi-\psi\|_{g} .
\end{aligned}
$$

This means that

$$
\left|\bar{f}_{i}(\phi)-\bar{f}_{i}(\psi)\right| \leq l_{i}\|\phi-\psi\|_{g}, \quad i=1, \ldots, n,
$$

for $l_{i}:=d_{i}^{-1} \sum_{j=1}^{n} l_{i j}(1+\delta) d_{j}$. Moreover, $\bar{b}_{i}$ satisfies (A2) with $\bar{\beta}_{i}=\beta_{i}$, and from (4.4) we have $\beta_{i}>l_{i}, i=1, \ldots, n$. The conclusion follows now from Theorem 3.2.

In order to apply the exponential stability criterion in Theorem 3.3 to model (4.1), we now assume that there exists a constant $\gamma>0$ such that all the normalized non-decreasing and bounded functions $\eta_{i j}^{(p)}$ in (4.1) satisfy

$$
\int_{-\infty}^{0} e^{-\gamma s} d \eta_{i j}^{(p)}(s)<\infty
$$

Theorem 4.3. Consider (4.1), where $a_{i}: \mathbb{R} \rightarrow(0,+\infty)$ and $b_{i}: \mathbb{R} \rightarrow \mathbb{R}$ are continuous, $f_{i j}^{(p)}, g_{i j}^{(p)}$ are Lipschitz functions with Lipschitz constants $\mu_{i j}^{(p)}, \sigma_{i j}^{(p)}$ respectively, and $\eta_{i j}^{(p)}$ are non-decreasing and bounded functions, normalized so that $\eta_{i j}^{(p)}(0)-\eta_{i j}^{(p)}(-\infty)=1, i, j=1, \ldots, n, p=1, \ldots, P$. Assume in addition that:

(i) (A2) is satisfied;

(ii) there exists a constant $\gamma>0$ such that $\eta_{i j}^{(p)}$ satisfy (4.7), $i, j=1, \ldots, n, p=1, \ldots, P$;

(iii) $a_{i}:=\inf \left\{a_{i}(x): x \in \mathbb{R}\right\}>0$ for $i=1, \ldots, n$.

If the matrix $N$ defined in (4.2) is a non-singular M-matrix, then there is a unique equilibrium point of (4.1), which is globally exponentially stable. 
Proof. Since $N$ is a non-singular M-matrix, there is $d=\left(d_{1}, \ldots, d_{n}\right)>0$ and $\delta>0$ such that (4.4) holds. We now claim that there is $\alpha \in(0, \gamma)$ such that

$$
\int_{-\infty}^{0} e^{-\alpha s} d \eta_{i j}^{(p)}(s)<1+\delta, \quad i, j=1, \ldots, n, p=1, \ldots, P .
$$

To prove the claim, we first note that, for each $\eta:=\eta_{i j}^{(p)}$ fixed, the function $F(t):=F_{i j}^{(p)}(t)=$ $\int_{-\infty}^{0} e^{-t s} d \eta_{i j}^{(p)}(s)$ is non-decreasing on $[0, \gamma]$. On the other hand, $F(\gamma)=\int_{-\infty}^{0} e^{-\gamma s} d \eta_{i j}^{(p)}(s)<\infty$ and $F(0)=\int_{-\infty}^{0} d \eta_{i j}^{(p)}(s)=1$. We now prove that $F$ is continuous on $[0, \gamma]$.

Fix $\varepsilon>0$. From (4.7), there is $M>0$ such that

$$
\int_{-\infty}^{-M} e^{-\alpha s} d \eta(s) \leq \int_{-\infty}^{-M} e^{-\gamma s} d \eta(s)<\varepsilon / 3, \quad \alpha \in[0, \gamma] .
$$

Since $f(\alpha, s)=e^{-\alpha s}$ is uniformly continuous on $[0, \gamma] \times[-M, 0]$, there is $\sigma>0$ such that $\mid e^{-\alpha s}-$ $e^{-\beta s} \mid \leq \varepsilon /(3 M)$ for all $s \in[-M, 0], \alpha, \beta \in[0, \gamma]$ with $|\alpha-\beta|<\sigma$, implying that

$$
\left|\int_{-M}^{0} e^{-\alpha s} d \eta(s)-\int_{-M}^{0} e^{-\beta s} d \eta(s)\right| \leq \varepsilon / 3
$$

Hence, we deduce that $|F(\alpha)-F(\beta)|<\varepsilon$, for $\alpha, \beta \in[0, \gamma]$ with $|\alpha-\beta|<\sigma$, proving the continuity of $F$. From the intermediate value theorem, it follows that for each $\eta_{i j}^{(p)}$ there is $\alpha_{i j}^{(p)} \in(0, \gamma)$ such that $\int_{-\infty}^{0} e^{-\alpha_{i j}^{(p)} s} d \eta_{i j}^{(p)}(s)<1+\delta$. Taking $\alpha=\min \left\{\alpha_{i j}^{(p)}: i, j=1, \ldots, n, p=1, \ldots, P\right\}$, then (4.8) holds.

Now, we argue as in the proof of Theorem 4.2 with $g(s)=e^{-\alpha s}$, and obtain that (A3) is fulfilled. The result follows from Theorem 3.3.

\section{Applications}

In this section, we shall apply the criteria in Section 4 to a large number of neural networks with infinite delay. The broad framework of our general results allows us to treat most of the neural network models considered in the literature (as well as some FDEs from population dynamics) as particular cases of the family of FDEs (3.1). Moreover, by presenting a comparison of results, we shall also show that our criteria improve in many cases those in recent papers.

We recall that the stability of a solution means stability relative to the set of solutions with initial conditions in $B C$.

Example 5.1. The cellular neural network with distributed delays

$$
\dot{x}_{i}(t)=-b_{i} x_{i}(t)+\sum_{j=1}^{n} f_{i j}\left(\int_{-\infty}^{0} x_{j}(t+s) d \eta_{i j}(s)\right), \quad i=1, \ldots, n,
$$

is a delayed version of the model (1.2) proposed by Hopfield [15], and a special case of (4.1). Here, $b_{i}>0, f_{i j}: \mathbb{R} \rightarrow \mathbb{R}$ are continuous and $\eta_{i j}:(-\infty, 0] \rightarrow \mathbb{R}$ are normalized, non-decreasing, and bounded functions. Applying Theorems 4.2 and 4.3 to this system, we have:

Corollary 5.1. Assume that $f_{i j}: \mathbb{R} \rightarrow \mathbb{R}$ are Lipschitz functions with Lipschitz constants $\mu_{i j}$ for $i, j=1, \ldots, n$. If

$$
N:=B-L, \quad \text { where } B=\operatorname{diag}\left(b_{1}, \ldots, b_{n}\right), L=\left[\mu_{i j}\right],
$$

is a non-singular M-matrix, then there is a unique equilibrium point of (5.1), which is globally asymptotically stable. Moreover, if there is $\gamma>0$ such that $\int_{-\infty}^{0} e^{-\gamma s} d \eta_{i j}(s)<\infty$ for $i, j=1, \ldots, n$, then the equilibrium is globally exponentially stable. 
Example 5.2. In [26], the following Cohen-Grossberg neural network model was studied:

$$
\dot{x}_{i}(t)=-a_{i}\left(x_{i}(t)\right)\left[b_{i}\left(x_{i}(t)\right)+\sum_{j=1}^{n} a_{i j} f_{j}\left(\int_{-\infty}^{0} k_{i j}(-s) x_{j}(t+s) d s\right)\right], \quad i=1, \ldots, n,
$$

where $a_{i j} \in \mathbb{R}, a_{i}: \mathbb{R} \rightarrow(0,+\infty)$ and $b_{i}: \mathbb{R} \rightarrow \mathbb{R}$ are continuous functions, $f_{j}: \mathbb{R} \rightarrow \mathbb{R}$ are Lipschitz functions, and the delay kernel functions $k_{i j}:[0, \infty) \rightarrow[0, \infty)$ are piecewise continuous and such that

$$
\int_{0}^{\infty} k_{i j}(s) d s=1
$$

In [26], the author established sufficient conditions for the global asymptotic stability of the equilibrium point of (5.2), assuming, as usual, bounded initial conditions.

If $P=1, f_{i j}^{(1)}(u)=a_{i j} f_{j}(u), g_{i j}^{(1)}(u)=u$ with $u \in \mathbb{R}$, and the bounded non-drecreasing functions $\eta_{i j}^{(1)}$ are of the form

$$
\eta_{i j}^{(1)}(s)=\int_{-\infty}^{s} k_{i j}(-u) d u, \quad s \in(-\infty, 0], \quad i, j=1, \ldots, n,
$$

then system (4.1) reduces to (5.2). Consequently, Theorem 4.2 applied to system (5.2) gives the following result:

Corollary 5.2. Consider (5.2), and, for $i, j=1, \ldots, n$, assume that:

(i) $b_{i}: \mathbb{R} \rightarrow \mathbb{R}$ satisfy (A2);

(ii) the positive kernels $k_{i j}$ satisfy (5.3);

(iii) $f_{i}: \mathbb{R} \rightarrow \mathbb{R}$ are $\mu_{i}$-Lipschitz functions;

(iv) the matrix $N=B-L$, where $B=\operatorname{diag}\left(\beta_{1}, \ldots, \beta_{n}\right)$ for $\beta_{i}$ as in (A2) and $L=\left[\left|a_{i j}\right| \mu_{j}\right]$, is a non-singular M-matrix.

Then there is a unique equilibrium $x^{*}$ of (5.2), which is globally asymptotically stable.

Remark 5.1. For system (5.2), L. Wang [26] obtained the existence and global asymptotic stability of an equilibrium point assuming the following hypotheses:

(a) For each $i \in\{1, \ldots, n\}, b_{i}$ is increasing and satisfies (A2);

(b) For each $i \in\{1, \ldots, n\}, f_{i}$ is bounded and $\mu_{i}$-Lipschitz continuous;

(c) For each $i \in\{1, \ldots, n\}$, there exist $\underline{a}_{i}, \bar{a}_{i}>0$ such that

$$
0<\underline{a}_{i} \leq a_{i}(u) \leq \bar{a}_{i}, \quad \forall u \in \mathbb{R} ;
$$

(d) For each $i, j \in\{1, \ldots, n\}$, the kernels $k_{i j}(t) \geq 0$ satisfy (5.3) and

$$
\int_{0}^{\infty} t k_{i j}(t) d t<\infty
$$

(e) The matrix $\underline{N}:=B \underline{A}-L^{T} \bar{A}$ is a non-singular M-matrix, where $B=\operatorname{diag}\left(\beta_{1}, \ldots, \beta_{n}\right), \underline{A}=$ $\operatorname{diag}\left(\underline{a}_{1}, \ldots, \underline{a}_{n}\right), \bar{A}=\operatorname{diag}\left(\bar{a}_{1}, \ldots, \bar{a}_{n}\right)$ and $L=\left[l_{i j}\right]$ with $l_{i j}=\left|a_{i j}\right| \mu_{j}$, for $\beta_{i}$ as in (A2), $i=1, \ldots, n$.

For $N=B-L$ as above, note that $\underline{N} \leq N^{T} \bar{A}$, and therefore if $\underline{N}$ is a non-singular M-matrix then $N^{T}$ is also a non-singular M-matrix, and $N$ as well [7]; however, the reverse is not true in general. It is clear that this set of assumptions is much more restrictive than the one assumed in Corollary 5.2 , which strongly improves the criterion in [26]. In particular, in this corollary no restrictions were imposed on the positive functions $a_{i}$.

Next, the application of Theorem 4.3 to (5.2) leads to sufficient conditions for its exponential stability, as follows: 
Corollary 5.3. Consider (5.2), and for $i, j=1, \ldots, n$ assume hypotheses (i)-(iv) in Corollary 5.2, and the additional conditions:

(v) there are constants $\underline{a_{i}}>0$ such that $0<\underline{a_{i}} \leq a_{i}(x)$ for all $x \in \mathbb{R}$;

(vi) for some $\gamma>0$, the positive kernels $k_{i j}$ satisfy

$$
\int_{0}^{\infty} k_{i j}(t) e^{\gamma t} d t<\infty, \quad i, j=1, \ldots, n
$$

Then, there is a unique equilibrium of (5.2), which is globally exponentially stable.

Remark 5.2. For system (5.2), Wu et al. [30] obtained the existence and global exponential stability of an equilibrium point under the conditions of $b_{i}$ differentiable with $b_{i}^{\prime}(x) \geq \beta_{i}$ for all $x \in \mathbb{R}$, for some $\beta_{i}>0$,

$$
\int_{0}^{\infty} k_{i j}(t) d t<1, \quad i, j=1, \ldots, n
$$

and the above hypotheses (iii), (vi), (c), (e). It is clear that the above Corollary 5.3 improves significantly the main result in [30].

Example 5.3. In [16], the authors studied the global asymptotic stability of the equilibrium point of the following Cohen-Grossberg neural networks model:

$\dot{x}_{i}(t)=-a_{i}\left(x_{i}(t)\right)\left[b_{i}\left(x_{i}(t)\right)+\sum_{j=1}^{n} a_{i j} f_{j}\left(x_{j}(t)\right)+\sum_{j=1}^{n} c_{i j} \int_{-\infty}^{0} k_{i j}(-s) g_{j}\left(x_{j}(t+s)\right) d s\right], i=1, \ldots, n,(5$

where $a_{i j}, c_{i j} \in \mathbb{R}, f_{j}, g_{j}: \mathbb{R} \rightarrow \mathbb{R}$ are Lipschitz functions and the kernels $k_{i j}(t)$ are non-negative and normalized, so that (5.3) holds, $i, j=1, \ldots, n$.

System (5.5) is another special case of model (4.1), when $P=2, f_{i j}^{(1)}(u)=a_{i j} f_{j}(u), g_{i j}^{(1)}(u)=u$, $f_{i j}^{(2)}(u)=c_{i j} u, g_{i j}^{(2)}(u)=g_{j}(u)$ with $u \in \mathbb{R}$, and the bounded variation functions $\eta_{i j}^{(1)}$ and $\eta_{i j}^{(2)}$ are of the form

$$
\eta_{i j}^{(1)}(s)=\left\{\begin{array}{ll}
1 & s=0 \\
0 & s<0
\end{array} \quad \eta_{i j}^{(2)}(s)=\int_{-\infty}^{s} k_{i j}(-u) d u, \quad s \in(-\infty, 0], \quad i, j=1, \ldots, n .\right.
$$

Consequently, from Theorems 4.2 and 4.3 we obtain the result:

Corollary 5.4. Assume (A2), $f_{j}, g_{j}: \mathbb{R} \rightarrow \mathbb{R}$ are Lipschitz functions with Lipschitz constant $\mu_{j}, \sigma_{j}$ respectively, and the non-negative kernels $k_{i j}$ satisfy (5.3) for all $i, j \in\{1, \ldots, n\}$. Suppose also that $N:=B-L$, where $B=\operatorname{diag}\left(\beta_{1}, \ldots, \beta_{n}\right)$ for $\beta_{i}$ as in (A2) and $L=\left[l_{i j}\right]$ with $l_{i j}=\left|a_{i j}\right| \mu_{j}+\left|c_{i j}\right| \sigma_{j}$, is a non-singular M-matrix.

Then there is a unique equilibrium $x^{*}$ of (5.5), which is globally asymptotically stable. Moreover, if the conditions (v) and (vi) in Corollary 5.3 hold, then $x^{*}$ is globally exponentially stable.

Remark 5.3. For system (5.5), T. Huang et al. [16] proved the global asymptotic stability of the equilibrium assuming in addition that, for each $i=1, \ldots, n, b_{i}$ is differentiable with $b_{i}^{\prime}(u) \geq \beta_{i}>0$, and that there exist $\underline{a}_{i}, \bar{a}_{i}>0$ such that $0<\underline{a}_{i} \leq a_{i}(u) \leq \bar{a}_{i}, u \in \mathbb{R}$. Thus the above Corollary 5.4 clearly improves the main result in [16].

Example 5.4. Consider the bidirectional associative memory (BAM) Cohen-Grossberg neural networks model

$$
\left\{\begin{array}{l}
\dot{x}_{i}(t)=-a_{i}\left(x_{i}(t)\right)\left[b_{i}\left(x_{i}(t)\right)+\sum_{j=1}^{m} f_{i j}\left(y_{j}\left(t-\tau_{i j}\right)\right)\right], \quad i=1, \ldots, n, \\
\dot{y}_{j}(t)=-d_{j}\left(y_{j}(t)\right)\left[c_{j}\left(y_{j}(t)\right)+\sum_{i=1}^{n} m_{j i} \int_{-\infty}^{0} k_{j i}(-s) g_{j i}\left(x_{i}(t+s)\right) d s\right], \quad j=1, \ldots, m,
\end{array}\right.
$$


where $m_{j i} \in \mathbb{R}, a_{i}, d_{j}: \mathbb{R} \rightarrow(0,+\infty)$ and $b_{i}, c_{j}: \mathbb{R} \rightarrow \mathbb{R}$ are continuous functions, $f_{i j}, g_{j i}: \mathbb{R} \rightarrow \mathbb{R}$ are Lipschitz functions and the kernels $k_{j i}:[0, \infty) \rightarrow[0, \infty)$ satisfy (5.3). For a description and explanation of the model, see $[3,17,18]$, also for further references.

The existence and global asymptotic stability of an equilibrium point of (5.6) were recently studied in [17]. As in the above examples, it is easy to see that (5.6) is a special case of model (4.1), thus from Theorem 4.2 we obtain the following result:

Corollary 5.5. For all $i=1, \ldots, n, j=1, \ldots, m$, assume that:

(i) $b_{i}(u)$ and $c_{j}(u)$ satisfy (A2) with constants $\beta_{i}>0$ and $\gamma_{j}>0$, respectively;

(ii) the kernels $k_{i j}(t)$ satisfy (5.3);

(iii) $f_{i j}, g_{j i}: \mathbb{R} \rightarrow \mathbb{R}$ are Lipschitzian and have Lipschitz constants $\mu_{i j}, \sigma_{j i}$, respectively;

(iv) $N$ is a non-singular M-matrix, where $N$ is defined by

$$
N:=\left[\begin{array}{cc}
B & -U \\
-S & G
\end{array}\right]_{(n+m) \times(n+m)},
$$

for

$$
B=\operatorname{diag}\left(\beta_{1}, \ldots, \beta_{n}\right), \quad G=\operatorname{diag}\left(\gamma_{1}, \ldots, \gamma_{m}\right), \quad U=\left[\mu_{i j}\right] \quad S=\left[\left|m_{j i}\right| \sigma_{j i}\right] .
$$

Then, there is a unique equilibrium of (5.6), which is globally asymptotically stable.

In [17], the authors assumed a different set of hypotheses to get the global asymptotic stability of the equilibrium point, since different norms in $\mathbb{R}^{n}$ were considered.

To the best of our knowledge, the global exponential stability of the BAM model (5.6) was never studied before. As an immediate consequence of Theorem 4.3, here we obtain the following criterion:

Corollary 5.6. Assume conditions (i)-(iv) in Corollary 5.5, and the additional hypotheses:

(v) there are $\underline{a}_{i}>0, \underline{d}_{j}>0$ such that $0<\underline{a}_{i} \leq a_{i}(u), 0<\underline{d}_{j} \leq d_{j}(u)$ for all $u \in \mathbb{R}$;

(vi) the kernels $k_{j i}$ satisfy (5.4), $i=1, \ldots, n, j=1, \ldots, m$.

Then the unique equilibrium of (5.6) is globally exponentially stable.

Example 5.5. Consider (4.1) with $P=2, a_{i}(u)=1, f_{i j}^{(1)}(u)=-a_{i j} f_{j}(u), g_{i j}^{(1)}(u)=u, f_{i j}^{(2)}(u)=$ $-b_{i j} u-I_{i}, g_{i j}^{(2)}(u)=g_{j}(u)$, for $u \in \mathbb{R}$, and $\eta_{i j}^{(1)}(s)=\left\{\begin{array}{ll}0, & s<0 \\ 1, & s=0\end{array}\right.$, and $\eta_{i j}^{(2)}(s)=\eta_{j}(s), i, j \in$ $\{1, \ldots, n\}$. Then, we obtain a model known as interval cellular neural network with S-type distributed delays (see [31]):

$$
\dot{x}_{i}(t)=-b_{i}\left(x_{i}(t)\right)+\sum_{j=1}^{n} a_{i j} f_{j}\left(x_{j}(t)\right)+\sum_{j=1}^{n} b_{i j} \int_{-\infty}^{0} g_{j}\left(x_{j}(t+s)\right) d \eta_{j}(s)+I_{i}, i=1, \ldots, n .
$$

Sufficient conditions for the existence and global exponential stability of an equilibrium of (5.7) are given from Theorem 4.3, and stated below.

Corollary 5.7. Assume that (A2) holds, $f_{i}, g_{i}: \mathbb{R} \rightarrow \mathbb{R}$ are Lipschitz functions with Lipschitz constants $\mu_{i}$ and $\sigma_{i}$ respectively, and the bounded variation functions $\eta_{i}:(-\infty, 0] \rightarrow \mathbb{R}$ are nondecreasing, normalized and satisfy

$$
\int_{-\infty}^{0} e^{-\gamma s} d \eta_{i}(s)<\infty, \quad i=1, \ldots, n
$$

for some $\gamma>0$. If

$$
N:=B-L, \quad \text { where } B=\operatorname{diag}\left(\beta_{1}, \ldots, \beta_{n}\right), L=\left[\left|a_{i j}\right| \mu_{j}+\left|b_{i j}\right| \sigma_{j}\right]
$$

is a non-singular M-matrix, then there is a unique equilibrium of (5.7), which is globally exponentially stable. 
Remark 5.4. For the particular system (5.7), R. Zhang \& L. Wang [31] obtained the existence and global exponential stability of an equilibrium assuming the general conditions on $f_{i}, g_{i}$ as above, and the following additional hypotheses:

(a) The functions $b_{i}$ are differentiable, with $\beta_{i}=\inf _{u \in \mathbb{R}} b_{i}^{\prime}(u)>0$;

(b) For each $i \in\{1, \ldots, n\}, \beta_{i}>\sum_{j=1}^{n}\left(\left|a_{j i}\right| \mu_{i}+\left|b_{j i}\right| \sigma_{i}\right)$.

Note that condition (b) implies that $N=B-\left[\left|a_{i j}\right| \mu_{j}+\left|b_{i j}\right| \sigma_{j}\right]$ is a diagonally dominant matrix, and therefore a non-singular M-matrix [7]. Hence, it is clear that the above corollary improves the main result in [31].

Neural networks (5.7) with bounded distributed delays were considered in [28] and [22]: in these papers, the bounded variation functions $\eta_{i j}(s)$ are zero on $(-\infty,-\tau]$, for some $\tau>0$. It is easy to see that the above results strongly improve the criteria established in [28] and [22], where only the global asymptotic stability of the equilibrium was addressed.

\section{Conclusions}

We have presented general criteria for the global asymptotic and global exponential stabilities of an equilibrium, for a large family of DDEs with infinite delay given here by equation (1.4). These criteria are simple to verify, do not involve the use of Lyapunov functionals, and are directly applicable to most of the autonomous neural network models with unbounded delay investigated in recent literature. All these models fall into the category of generalized Cohen-Grossberg neural networks.

This work complements that of $[6,22]$, where the situation of neural networks with distributed bounded delay was considered. In general, the introduction of large delays in systems of differential equations is not harmless. It can produce oscillations, loss of stability of equilibria, existence of unbounded solutions. For the situation of infinite delay, typically the "memory functions" appear as integral kernels, diminish when going back in time, and finally disappear at $-\infty$. Roughly speaking, in this paper the results on the global stability of an equilibrium have been obtained by assuming that the instantaneous negative feedback terms dominate the delay effect, so that in spite of the unbounded delays, the DDE behaves similarly to an ODE.

As illustration, we have applied our general results to a significant number of concrete CohenGrossberg neural networks, and provided immediate sufficient conditions for their global stability. Since Volterra's works, it is well known that the global stability of ODE systems which serve as models in population dynamics is strongly related to the algebraic properties of the so-called competition matrix, whose entries reflect the relationships among species, determined by the response of the growth rate of each species to the increase of each other. This has led to the concept of M-matrix, or matrices with similar algebraic properties. As usual, in order to show the global attractivity of an equilibrium for DDEs modelling neural networks, here we have required that the network "connection" matrix is a non-singular M-matrix. This means that the instantaneous self-connections overpower the connections among neurons. To obtain the global exponential stability, we have further assumed that the memory functions have a kind of exponential decay at $-\infty$.

We have also discussed and compared our results with those of other authors, showing the advantage of our method. Not only have we often obtained better criteria, but also our general approach applies directly to all autonomous generalized Cohen-Grossberg neural models. In contrast, the results on global stability for delayed neural networks in the literature are usually obtained by considering a specific Lyapunov functional for each particular model under study.

In a forthcoming work, we shall exploit the ideas beyond our general method to address the global attractivity of equilibria for non-autonomous neuron networks with unbounded time-dependent discrete delays.

Acknowledgments. This research was supported by FCT (Portugal), through Financiamento Base 2009-ISFL-1-209 (Teresa Faria) and the research center CMAT (José J. Oliveira). 


\section{References}

[1] J. Bélair, Stability in a model of a delayed neural network, J. Dynam. Differential Equations 5 (1993) 607-623.

[2] S.A. Campbell, Delay independent stability for additive neural networks, Diff. Eqns. Dyn. Syst. 9 (2001) 115-138.

[3] M. Cohen and S. Grossberg, Absolute stability, global pattern formation,parallel memory storage bycompetitive neural networks, IEEE Trans. Systems Man Cybernet. 13 (1983) 815-826.

[4] T. Faria, Stability and Extinction for Lotka-Volterra systems with Infinite Delay, J. Dynam. Differential Equations 22 (2010), 299-324.

[5] T. Faria and J.J. Oliveira, Local and global stability for Lotka-Volterra systems with distributed delays and instantaneous negative feedbacks, J. Differential Equations 244 (2008), 1049-1079.

[6] T. Faria and J.J. Oliveira, Boundedness and global exponential stability for delayed differential equations with applications, Appl. Math. Comput. 214 (2009), 487-496.

[7] M. Fiedler, Special Matriz and Their Applications in Numerical Mathematics, Martinus Nijhoff Publ. (Kluwer), Dordrecht, 1986.

[8] M. Forti and A. Tesi, New condition for global stability of neural networks with application to linear, quadratic programming problems, IEEE Trans. Circuits Syst. 42 (1995) 354-366.

[9] K. Gopalsamy and X.Z. He, Stability in asymmetric Hopfield nets with transmission delays, Physica D 76 (1994), 344-358.

[10] K. Gopalsamy and I. Leung, Delay induced periodicity in a neural netlet of excitation and inhibition, Physica D 89 (1996), 395-426.

[11] J. Haddock and W. Hornor, Precompactness and convergence in norm of positive orbits in a certain fading memory space, Funkcial. Ekvac. 31 (1988) 349-361.

[12] J.R. Haddock, M.N. Nkashama, J. Wu, Asymptotic Constancy for Linear Neutral Volterra Integrodifferential Equations, Tôhoku Math. J. 41 (1989) 689-710.

[13] J.K. Hale and J. Kato, Phase Space for Retarded Equations with Infinite Delay, Funkcial Ekvac, 21 (1978) 11-41.

[14] J.J. Hopfield, Neural networks and physical systems with emergent collective computational abilities, Proc. Nat. Acad. Sci. U.S.A. 79 (1982), 2554-2558

[15] J.J. Hopfield, Neural networks with graded response have collective computational properties like those of two-state neurons, Proc. Nat. Acad. Sci. 81 (1984) 3088-3092.

[16] T. Huang, C. Li and G. Chen, Stability of Cohen-Grossberg neural networks with unbounded distributed delays, Chaos Solitons \& Fractals, 34 (2007) 992-996.

[17] H. Jiang and J. Cao, BAM-type Cohen-Grossberg neural networks with time delay, Math. Comput. Modelling 47 (2008) 92-103.

[18] B. Kosko, Bidirectional associative memories, IEEE Trans. Systems Man Cybernet. 18 (1988) 49-60.

[19] J. Liang and J. Cao, Global asymptotic stability of bi-directional associative memory networks with distributed delays, Appl. Math. Comput. 152 (2004), 415-424.

[20] C.M. Marcus and R.M. Westervelt, (1989), Stability of analogy neural networks with delay, Physics Reviews A 39, 347-359. 
[21] L. Olien and J. Bélair, Bifurcations, stability, and monotonicity properties of a delayed neural network model, Physica D 102 (1997) 349-363.

[22] J.J. Oliveira, Global asymptotic stability for neural network models with distributed delays, Math. Comput. Modelling 50 (2009) 81-91.

[23] S. Ruan and R. Filfil, Dynamics of a two-neuron system with discrete and distributed delays, Physica D 191 (2004) 323-342.

[24] Q. Song and J. Cao, Stability analysis of Cohen-Grossberg neural network with both time-varying and continuously distributed delays, J. Comput. Appl. Math. 197 (2006) 188-203.

[25] P. van den Driessche and X. Zou, Global stability in delayed hopfield neural network models, SIAM J. Appl. Math. 58 (1998) 1878-1890.

[26] L. Wang, Stability of Cohen-Grossberg neural networks with distributed delays, Appl. Math. Comput. 160 (2005) 93-110.

[27] L. Wang, X. Zou, Hopf bifurcation in bidirectional associative memory neural networks with delays: analysis and computation, J. Comput. Appl. Math. 167 (2004) 73-90.

[28] M. Wang and L. Wang, Global asymptotic stability of static neural networks with S-type delays, Math. Comput. Modelling 44 (1)(2006) 218-222.

[29] J. Wu, Symmetric functional-differential equations and neural networks with memory, Trans. Amer. Math. Soc. 350 (1998), 4799-4838.

[30] L. Wu, B.T. Cui and X.Y. Lou, Global exponential stability of Cohen-Grossberg neural networks with distributed delays, Math. Comput. Modelling 47 (2008) 868-873.

[31] R. Zhang and L. Wang, Global exponential robust stability of interval cellular neural networks with S-type with distributed delays, Math. Comput. Modelling 50 (2009) 380-385. 